\title{
Induced radioactivity studies of the shielding and beamline equipment of the high intensity proton accelerator facility at PSI
}

\author{
Polina Otiougova $^{\mathrm{a}}$, Ryan Bergmann, Daniela Kiselev, Vadim Talanov, and Michael Wohlmuther \\ Paul Scherrer Institute (PSI), 5232 Villigen, Switzerland
}

\begin{abstract}
The Paul Scherrer Institute (PSI) is the largest national research center in Switzerland. Its multidisciplinary research is dedicated to a wide field in natural science and technology as well as particle physics. The High Intensity Proton Accelerator Facility (HIPA) has been in operation at PSI since 1974. It includes an $870 \mathrm{keV}$ Cockroft-Walton pre-accelerator, a $72 \mathrm{MeV}$ injector cyclotron as well as a $590 \mathrm{MeV}$ ring cyclotron. The experimental facilities, the meson production graphite targets, Target $\mathrm{E}$ and Target $\mathrm{M}$, and the spallation target stations (SINQ and UCN) are used for material research and particle physics. In order to fulfill the request of the regulatory authorities and to be reported to the regulators, the expected radioactive waste and nuclide inventory after an anticipated final shutdown in the far future has to be estimated. In this contribution, calculations for the $20 \mathrm{~m}$ long beamline between Target $\mathrm{E}$ and the $590 \mathrm{MeV}$ beam dump of HIPA are presented. The first step in the calculations was determining spectra and spatial particle distributions around the beamlines using the Monte-Carlo particle transport code MCNPX2.7.0 [1]. To perform the analysis of the MCNPX output and to determine the radionuclide inventory as well as the specific activity of the nuclides, an activation script [2] using the FISPACT10 code with the cross sections from the European Activation File (EAF2010) [3] was applied. The specific activity values were compared to the currently existing Swiss exemption limits (LE) [4] as well as to the Swiss liberation limits (LL) [5], becoming effective in the near future. The obtained results were used to estimate the total volume of the radioactive waste produced at HIPA and have to be reported to the Swiss regulatory authorities. The comparison of the performed calculations to measurements is discussed as well.
\end{abstract}

\section{Introduction}

According to the request of the Swiss Authorities, the volume of the radioactive waste expected to be produced at the High Intensity Proton Accelerator Facility (HIPA) of the Paul Scherrer Institute (PSI) was calculated. The study was aiming to assess the volume of radioactive material produced by the facility after its final shutdown. Year 2058 was used as a reference time for the calculation. In this publication the activation of the $20 \mathrm{~m}$ long beamline and its surrounding shielding between the Target $\mathrm{E}$ and beam dump is discussed. This includes:

- Particle transport from the loss points of the $590 \mathrm{MeV}$ proton beam to the surrounding shielding by Monte-Carlo (MC) simulation employing the particle transport code MCNPX2.7.0 [1];

- Combination of the MC simulation via the Activation Script [2] with SP-FISPACT10 code and the European Activation Files (EAF2010) [3] in order to obtain radionuclide inventories.

As the radioactive material criterion, the clearance index calculated according to the summation rule was defined:

$$
C I=\sum_{i=1} \frac{A_{i}}{C V_{i}}
$$

where $A_{i}$ is a nuclide specific activity and $C V_{i}$ is a nuclide clearance value. For the clearance values for each

a e-mail: Polina.Otiougova@psi.ch nuclide the LE (exemption limits) values from [4] and LL (liberation limits) from [5] were considered. Starting from year 2018 the LL values are supposed to substitute the currently valid LE values in the Swiss radiation protection regulations. It has to be noted that the natural radionuclides like ${ }^{40} K$, Uranium, Thorium and daughters were not included in the overall clearance index calculation. The clearance values for the natural nuclides are significantly higher than the ones for the artificially created nuclides. For this reason their influence on total waste volume is not significant. The radioactive waste volume was calculated for the following decay times, after operation until 2058:

- 10 years +30 years: first 10 years are considered to be the decommissioning planning time (post-closure phase);

- 10 years +75 year;

- 10 years +100 years.

The irradiation history of the beamline was divided into two parts. The first one corresponds to the irradiation of the region between the Target $\mathrm{E}$ and the magnet bending the proton beam to SINQ (AHL-Magnet). The second part of the simulation considered the region downstream of the AHL-Magnet up to the beam dump. The values of the assumed irradiation history are summarized in the Table 1.

\section{Details of the simulation}

The MCNPX2.7.0 code was applied to obtain the neutron spectra for each cell of the model. 315 neutron

(C) The Authors, published by EDP Sciences. This is an Open Access article distributed under the terms of the Creative Commons Attribution License 4.0 (http://creativecommons.org/licenses/by/4.0/). 
Table 1. The irradiation history of the beamline.

\begin{tabular}{lccc}
\hline Years & $1974-1990$ & $1991-2013$ & $2014-2058$ \\
\hline $\begin{array}{l}\text { TgE }- \\
\text { AHL-Magnet }\end{array}$ & Negligible & $6.79 \mathrm{Ah}$ & $10 \mathrm{Ah}$ \\
$\begin{array}{l}\text { AHL-Magnet }- \\
\text { Beam dump }\end{array}$ & Negligible & $0.35 \mathrm{Ah}$ & $0.35 \mathrm{Ah}$ \\
\hline
\end{tabular}

energy groups were used to obtain the neutron fluxes up to $20 \mathrm{MeV}$. The rnucs-card [6] was used in the calculation to include the contribution of the spallation products produced in the "model regime" of MCNPX. A realistic geometrical representation of the beamline elements as well as the beamline shielding was build. Due to the enormous computation time required to obtain good statistics inside several meters of shielding, weight windows had to be used used as variance reduction technique.

\subsection{Geometry model}

The biological radiation shielding of the beamline and the AHL Magnet (the main magnet of the Beamline) were initially modeled in CATIA-V5 [7]. In order to simplify the initial model and to implement it into MCNPX, it was modified in SpaceClaim, a CAD tool provided by ANSYS [8]. In order to convert the SpaceClaim STEP file, the multi-physics coupling analysis modeling program (SuperMC/MCAM) developed by the Institute of Nuclear Energy Safety Technology (INEST) and the CASFDS Team [9] was used. The MCNPX geometry model including materials and beamline elements is depicted in Fig. 1 and Fig. 2. It should be mentioned that the first $2.5 \mathrm{~m}$ of the second stage concrete and steel shielding around the beamline was subdivided into $25 \mathrm{~cm}$ wide slices. This was performed in order to adjust averaging of the neutron flux. For the reason of neutron flux attenuation in the concrete, averaging in a large volume should be avoided, since it could lead to large uncertainties in the radioactive waste volume estimation. The slicing of the concrete and steel shielding elements around the beamline can be seen in Figs. 1 and 2 as well. The inner most part of the steel shielding surrounding the beamline was not sliced because the activation of the materials is high in this region; all the elements in this region of the shielding contributed to the radioactive waste volume.

\subsection{Materials}

Since some of the trace elements and impurities contained in the materials have high neutron capture cross sections, the elemental composition of each material is particularly important. The content of impurities in the main materials is summarized in the Table 2. For the concrete, the Europium content was measured using the Neutron Activation Analysis (NAA) Method; therefore, several concrete samples were irradiated in a dedicated irradiation station of the spallation source SINQ at PSI. In order to account for gaps between shielding elements, effective densities of $7.4 \mathrm{~g} / \mathrm{cm}^{3}$ and $2.4 \mathrm{~g} / \mathrm{cm}^{3}$ were used for steel and concrete respectively.
Table 2. Impurity content in the main materials.

\begin{tabular}{lll}
\hline Element & \multicolumn{1}{c}{ Steel } & Concrete \\
\hline $\mathrm{Co}$ & $170 \mathrm{ppm}$ & $5 \mathrm{ppm}$ \\
$\mathrm{Eu}$ & $1 \mathrm{ppm}$ & $0.5 \mathrm{ppm}$ \\
$\mathrm{Th}$ & $0.5 \mathrm{ppm}$ & $1 \mathrm{ppm}$ \\
$\mathrm{U}$ & $1 \mathrm{ppm}$ & $1.5 \mathrm{ppm}$ \\
\hline
\end{tabular}

\subsection{Biasing}

In order to achieve statistically relevant results in thick shielding, biasing techniques were required. The buildin MCNPX methods were either too time consuming or did not provide the necessary speed up of the simulation. Hence, the following technique was used for the weight window generation:

- Define a spatial mesh to calculate neutron fluxes for the full geometry;

- Define energy binning on neutron flux: from thermal to high energies. Energy bins in $\mathrm{MeV}: 10^{-4}-10^{-2}, 10^{-2}-$ $1.0,1.0-20.0,20.0-600.0$

- The neutron flux was converted into weight windows (the inverse of importance) [10].

It has to be mentioned that the density reduction for factor 10 was applied to simplify the particle propagation during the runs for the weight windows generation.

\subsection{Postprocessing}

After obtaining the neutron fluxes and radionuclide production rates with MCNPX, the Activation Script was used to determine the radionuclide inventory. This script runs the SF-FISPACT10 code using the EAF 2010 cross sections and irradiation history in order to obtain the nuclide inventory of each cell at any time step. For the radioactive waste volume calculations the clearance indecies are evaluated for each cell and all cells with $C I>1$ contribute to the waste volume. The sum of the radioactive cells volume of each materials was calculated using an in-house developed python script.

\section{Results}

As a final outcome of the study, the radioactive waste volume for each material was summed and so was the nuclide inventory of the cells with the same materials. The volume was estimated with both the LE and LL clearance values.

\subsection{The waste volume}

The volume of the radioactive waste after operation until 2058 was calculated for current LE values as well as the future LL values. An increase in the waste volume in case of LL values was observed as expected. The increase of the volume corresponds to a factor 1.4 for steel and for a factor of 4.9 for concrete assuming 10 years +30 years cooling time after 2058. However, it has to be mentioned that these factors have large variations, when evaluating the waste volumes in the other regions of HIPA. The radionuclide inventory for concrete and steel including the nuclides with specific activity higher than $1.0 \mathrm{~Bq} / \mathrm{g}$ is shown in the Table 3, using the future clearance values LL. 


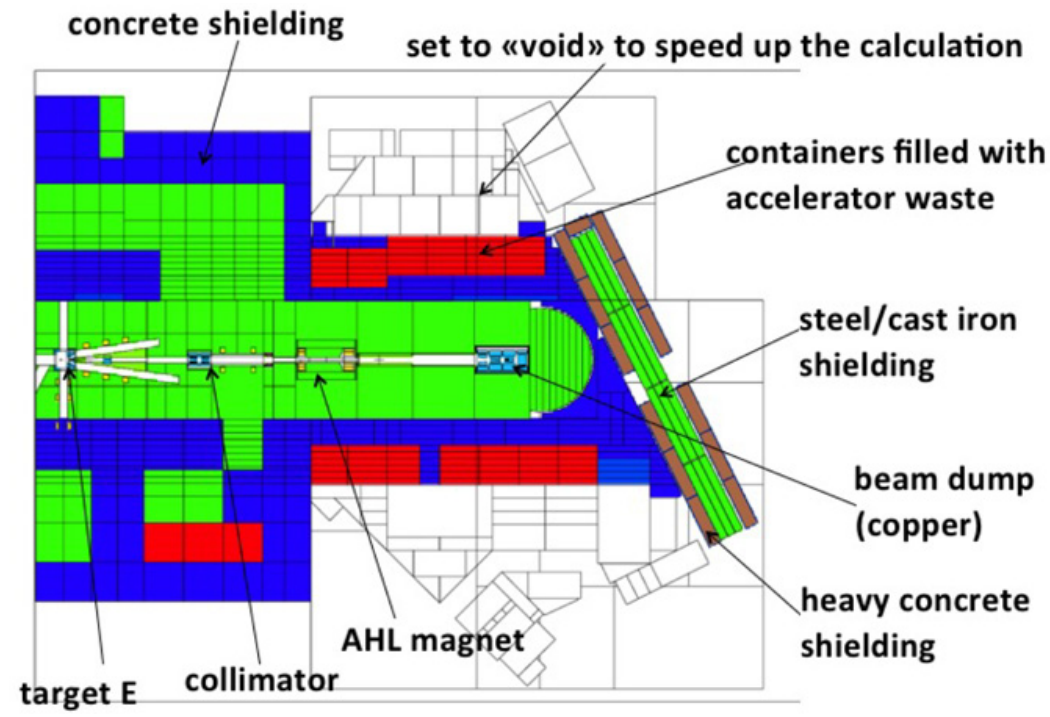

Figure 1. Horizontal cut through the MCNPX geometry model, XZ=Plane. Different colors represent different materials used in the beamline and shielding; green: steel/cast iron, blue: concrete, light blue and yellow: copper, red: waste containers with accelerator waste, brown: heavy concrete.

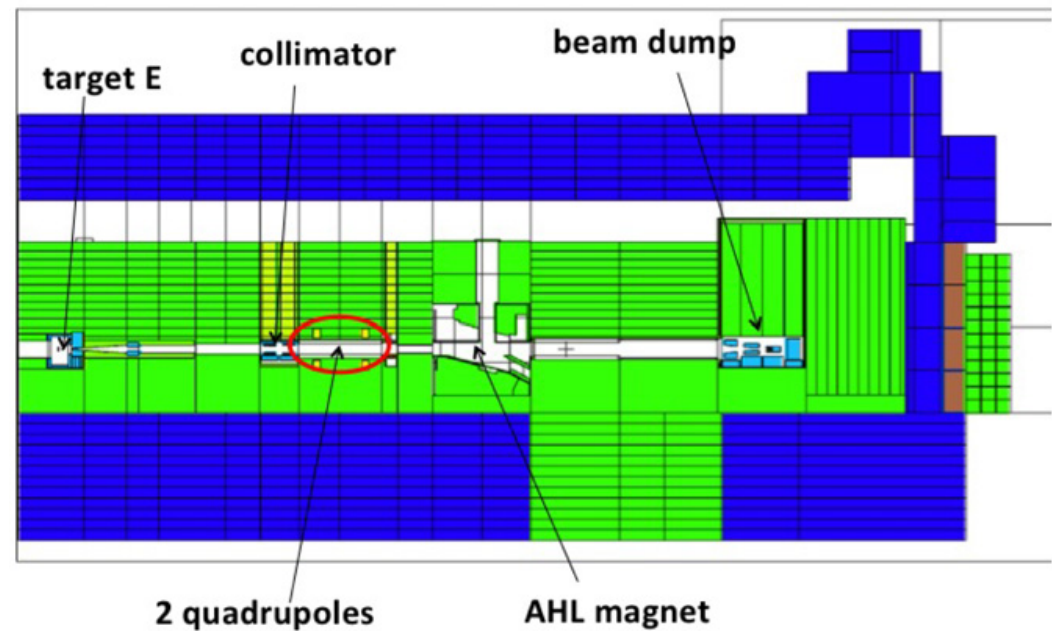

Figure 2. Vertical cut through the MCNPX geometry model, YZ=Plane. The major beamline elements are labeled. Each color indicates a material type; the color code is the same as in Fig. 1.

Table 3. Radionuclide inventory for steel and concrete. Operation until 2058 and cooling time $10 \mathrm{y}+30 \mathrm{y}$, future LL clearance values applied. Only the nuclides $\mathrm{A}_{s}>1 \mathrm{~Bq} / \mathrm{g}$ are listed. In the table $\mathrm{A}_{s}$ stands for specific activity.

\begin{tabular}{lll}
\hline & Steel & Concrete \\
\hline Nuclide & $\mathrm{A}_{s}(\mathrm{~Bq} / \mathrm{g})$ & $\mathrm{A}_{s}(\mathrm{~Bq} / \mathrm{g})$ \\
\hline $\mathrm{H}-3$ & 420.5 & 322.0 \\
$\mathrm{C}-14$ & $<1$ & 4.38 \\
$\mathrm{Ar}-39$ & 40.4 & 2.7 \\
$\mathrm{Ar}-42$ & 3.8 & $<1$ \\
$\mathrm{Ca}-41$ & $<1$ & 16.4 \\
$\mathrm{Fe}-55$ & 19.6 & $<1$ \\
$\mathrm{Co}-60$ & 262.2 & $<1$ \\
$\mathrm{Ni}-59$ & 4.7 & $<1$ \\
$\mathrm{Ni}-63$ & 323.2 & $<1$ \\
$\mathrm{Nb}-93 \mathrm{~m}$ & 3.5 & $<1$ \\
$\mathrm{Mo}-93$ & 2.34 & $<1$ \\
Eu-152 & 111.1 & 18.6 \\
Eu-154 & 31.4 & $<1$ \\
\hline
\end{tabular}

\subsection{Comparison with measurements}

The simulated specific activities of the most important isotopes produced in concrete were compared with measurements performed on the concrete sample placed on the top of the radiation shielding straight above the Target E. The sample was extracted from the beamline in March 2016 and the dedicated gamma-spectrometry measurements were performed on 05.04.16 [11]. In order to compare the results, the geometry model of the simulation was extended with a model of a concrete sample above the steel shielding of the beamline. The irradiation history and cooling time were adjusted respectively. The results of the comparison are shown in the Table 4.

It should be noted, that the simulated values for the main nuclides (Co-60, Eu-152, Eu-154) exceed the measured ones by a factor 3 to 4 resulting in a conservative estimate for the radioactive waste volume. The reason of this somehow systematic discrepancy can be explained by the possible variations between the simulated and the real 
Table 4. Measured specific activities in the concrete sample and comparison to calculation [11].

\begin{tabular}{lcc}
\hline Nuclide & Measured $\mathrm{A}_{s}(\mathrm{~Bq} / \mathrm{g})$ & Simulated $\mathrm{A}_{s}(\mathrm{~Bq} / \mathrm{g})$ \\
\hline $\mathrm{Na}-22$ & 1.6 & 7.44 \\
$\mathrm{Fe}-59$ & 0.36 & 0.72 \\
$\mathrm{Co}-60$ & 4.22 & 14.8 \\
$\mathrm{Zn}-65$ & 0.69 & 1.57 \\
$\mathrm{Cs}-134$ & 3.17 & 4.18 \\
Eu-152 & 6.07 & 23.2 \\
Eu-154 & 1.0 & 3.27 \\
\hline
\end{tabular}

experimental conditions of activation of the sample. This includes particulary the approximations in position of the sample and in the geometry model.

\subsection{HIPA facility}

Almost the complete $590 \mathrm{MeV}$ area of the HIPA facility including the Ring Cyclotron, Target $\mathrm{M}$ region, SINQ and UCN spallation targets was modeled and the radioactive waste volume and the nuclide inventory were calculated using a similar method as described above. The $72 \mathrm{MeV}$ region as well as some other parts have still to be calculated in the future.

\section{References}

[1] D. Pelowitz, MCNPX User's Manual Version 2.7.0, Los Alamos National Laboratory, LA-CP-11-00438, 2011
[2] Franz X. Gallmeier and Michael Wohlmuther, "ACTIVATION SCRIPT VERSION 1.0 USER GUIDE," ORNL/TM-2008/031 (August 18, 2008)

[3] J.-Ch. Sublet, L.W. Packer, J. Kopecky, R.A. Forrest, A.J. Koning and D.A. Rochman, The European Activation File: EAF-2010 neutron- induced cross section library. EASY Documentation Series, CCFER (10) 05

[4] Schweizerische Strahlenschutzverordnung, 01.01. 2014

[5] Draft version of the "Anhang 3 Strahlenschutzverordnung”, Mail from N. Stritt (BAG), 05.02.2015

[6] Franz X. Gallmeier and Michael Wohlmuther, The Residual Nuclei Patch of MCNPX 2.6.0 and 2.7.0; Paul Scherrer Insitute (PSI), TM-85-15-03

[7] CATIA-V5, Dassault Systmes, http://www.3ds. com/products-services/catia/products/

[8] ANSYS SpaceClaim Direct Modeler, http://www . ansys.com/Products

[9] Y. Wu, FDS Team, CAD-based interface programs for fusion neutron transport simulation, Fusion Engineering and Design 84, 1987-1992 (2009); Wu Yican, Song Jing, Zheng Huaqing, et al. CAD-Based Monte Carlo Program for Integrated Simulation of Nuclear System SuperMC, Annals of Fusion Energy, doi:10.1016/j.anucene.2014.08.058

[10] Solomon, C.J., Sood, A., \& Booth, T.E. (2009). A weighted adjoint source for weight-window generation by means of a linear tally combination. United States: American Nuclear Society - ANS

[11] S. Heinitz, gamma-spectrometry report, Paul Scherrer Institute (PSI), 05.04.2016 